Eric S. Key, Department of Mathematical Sciences, University of Wisconsin-Milwaukee, Milwaukee, Wisconsin 53201, USA, e-mail:

ericskey@csd.uwm.edu

\title{
SYMMETRIC MEASURE-PRESERVING SYSTEMS
}

\begin{abstract}
A symmetric measure-preserving system is one where the measure $\operatorname{Pr}$ is preserved by two maps $T$ and $R$ where $R$ is self-inverse and $T \circ R=T$. We discuss the existence of such systems and some consequences, including when unimodal maps are conjugate to the symmetric tent map.
\end{abstract}

\section{Introduction}

A continuous map $T:[0,1] \rightarrow[0,1]$ is called unimodal with turning point $m$ if $m \in(0,1)$ and $T$ is continuous, strictly increasing on $[0, m]$ and strictly decreasing on $[m, 1]$. For the moment, let us call a unimodal map two-to-one if $T(0)=T(1)=0$ and $T(m)=1$. To each two-to-one map we can associate a unique continuous map $R:[0,1] \rightarrow[0,1]$ such that $R$ is not the identity and $T \circ R=T$. The most well-known such pair of maps is $\tau(x)=\min (2 x, 2(1-x))$ and $\rho(x)=1-x$.

For each probability measure $\operatorname{Pr}$ on the Borel subsets of $[0,1]$ we may define the function $F:[0,1] \rightarrow[0,1]$ defined by $F(t)=\operatorname{Pr}([0, t])$. We will call $F$ the distribution function associated with $\mathrm{Pr}$.

Given a two-to-one map $T$ one problem of interest is to characterize the probability measures $\operatorname{Pr}$ which are preserved by $T$. It is well-known that such measures exist. In the case of $\tau$ we know that Lebesgue measure on $[0,1]$ is one such probability measure. We also note that this measure is preserved by $\rho$.

Suppose for a moment that given a two-to-one map $T$ with turning point $m$ and its associated map $R$ that we can find a probability measure Pr which

Key Words: Measure-preserving, symmetric tent map

Mathematical Reviews subject classification: Primary: 28D05. Secondary: 47A35

Received by the editors July 28, 1997 
is preserved by both $T$ and $R$. Let $F$ be the distribution function associated with $\operatorname{Pr}$. Since $R$ preserves $\operatorname{Pr}$ we have

$$
F(R(t))=1-F(t)=\rho(F(t)),
$$

and since $T$ preserves $\operatorname{Pr}$, for $x \in[0, m]$ we have

$$
F(T(x))=F(x)+1-F(R(x))=2 F(x)=\tau(F(x)),
$$

while for $x \in[m, 1]$ we have

$$
F(T(x))=F(T(R(x)))=\tau(F(R(x))=\tau(\rho(F(x)))=\tau(F(x)) .
$$

Thus we have

$$
\begin{aligned}
& F \circ R=\rho \circ F \\
& F \circ T=\tau \circ F
\end{aligned}
$$

Note that to this point we only use symmetry. Suppose in addition we know that $F$ is strictly increasing and continuous. Such would be the case if the probability measure was non-atomic and assigned positive probability to all sub-intervals of $[0,1]$. In this case we have

$$
\begin{aligned}
& R=F^{-1} \circ \rho \circ F \\
& T=F^{-1} \circ \tau \circ F,
\end{aligned}
$$

and we would have proven that $T$ and $\tau$ (and $R$ and $\rho$ ) are topologically conjugate.

Conversely, suppose that $T$ is two-to-one with turning point $m, R$ is the associated map with $T \circ R=T$, and for some homeomorphism $F:[0,1] \rightarrow[0,1]$ we have $F \circ T=\tau \circ F$ and $F \circ R=\rho \circ F$. Then $F$ is the distribution function of a probability measure preserved by both $T$ and $R$. To see why, let $\operatorname{Pr}$ be the probability measure on the Borel subsets of $[0,1]$ whose distribution function is $F$. Such Pr exists by the Carathéodory extension theorem. It is sufficient to check that the measures of intervals of the form $[0, y]$ are preserved. Since $T$ is two-to-one there is a unique $x \in[0, m]$ with $T(x)=y$, and we have $T^{-1}([0, y])=[0, x] \cup[R(x), 1]$, and $R^{-1}([0, y])=[R(y), 1]$. Note that $R(m)=m$ so that $F(m)=F(R(m))=\rho(F(m))=1-F(m)$ so $F(m)=1 / 2$. Hence

$$
\begin{aligned}
\operatorname{Pr}([0, x] \cup[R(x), 1]) & =F(x)+1-F(R(x))=F(x)+\rho(F(R(x)) \\
& =2 F(x)=\tau(F(x))=F(T(x))=\operatorname{Pr}([0, y]),
\end{aligned}
$$


and

$$
\operatorname{Pr}([R(y), 1])=1-F(R(y))=\rho(F(R(y))=F(y)=\operatorname{Pr}([0, y]) .
$$

In this paper we

1. Generalize the idea of two-to-one maps to abstract measure spaces.

2. In the case where the measure space is a compact metric space, show that there are non-atomic probability measures preserved by two-to-one maps (suitably defined) which are also preserved by the reflection map $R$.

3. In the case where the compact metric space is $[0,1]$, give conditions on two-to-one maps which ensure that this measure will give positive probability to any subinterval of $[0,1]$.

4. In the case of $[0,1]$, look at what happens if we have non-atomic probability measures which give probability 0 to some subintervals of $[0,1]$.

\section{Some Additional Definitions and Examples}

We will call the quintuple $(\Omega, \mathcal{F}, \operatorname{Pr}, T, R)$ a symmetric measure-preserving system if

P0: $(\Omega, \mathcal{F}, \operatorname{Pr})$ is a probability space;

P1: $(\Omega, \mathcal{F}, \operatorname{Pr}, T)$ is a measure-preserving system;

P2: $(\Omega, \mathcal{F}, \operatorname{Pr}, R)$ is a measure-preserving system;

P3: $\{\omega \in \Omega: R(\omega) \neq \omega\} \in \mathcal{F}$ and $\operatorname{Pr}(\{\omega \in \Omega: R(\omega) \neq \omega\})>0$;

P4: $R(R(\omega))=\omega$ for all $\omega \in \Omega$;

P5: $T \circ R=T$.

We shall call a measurable map $R$ of $(\Omega, \mathcal{F}, \operatorname{Pr})$ a reflection of $(\Omega, \mathcal{F}, \operatorname{Pr})$ if it satisfies (P3) and (P4). If we have no measure in mind, we shall call a measurable map $R$ of $(\Omega, \mathcal{F})$ a reflection of $(\Omega, \mathcal{F})$ if $R$ is not the identity map and $R \circ R$ is the identity map.

Two examples of symmetric measure-preserving systems are

- $\Omega=[0,1]$;

- $\mathcal{F}=$ the Borel subsets of $[0,1]$; 
- $\operatorname{Pr}(E)=$ the ordinary Lebesgue measure of $E$;

- $T(x)=\min (2 x, 2(1-x))$;

- $R(x)=1-x$

and

- $\Omega=[0,1]$;

- $\mathcal{F}=$ the Borel subsets of $[0,1]$;

- $\operatorname{Pr}(E)=\int_{E} \frac{1}{\pi \sqrt{x-x^{2}}} d x$

- $T(x)=4 x(1-x)$;

- $R(x)=1-x$.

Note that the probability measure in the second example is non-atomic and gives positive probability to all subintervals of $[0,1]$. This provides one example of the situation discussed in the previous section.

We now proceed to generalize our earlier idea of two-to-one. Note that we drop the requirement that the map be onto.

Suppose that $\mathcal{F}$ is a $\sigma$-algebra on the set $\Omega$ and that $T$ is a measurable map from $\Omega$ to $\Omega$. We shall say that $T$ is two-to-one if there are measurable sets $\Omega_{l}$ and $\Omega_{r}$ and a reflection $R$ of $(\Omega, \mathcal{F})$ with the properties that

- $\Omega=\Omega_{l} \cup \Omega_{r}$;

- $\Omega_{l} \cap \Omega_{r}$ is the set of fixed points of $R$;

- $T \circ R=T$;

- The restriction of $T$ to each of $\Omega_{l}$ and $\Omega_{r}$ is one-to-one;

- If $F \in \mathcal{F}$ then $T\left(F \cap \Omega_{l}\right) \in \mathcal{F}$ and $T\left(F \cap \Omega_{r}\right) \in \mathcal{F}$.

Since we can show that there is exactly one such $R$ for any two-to-one map $T$, we will refer to $R$ as the reflection associated with $T$. Also note that the sets $\Omega_{l}$ and $\Omega_{r}$ cannot be empty and that $R$ maps each of these sets onto the other. Two-to-one maps are a natural generalization of unimodal maps.

We have seen examples of two-to-one maps on $[0,1]$. Here are some examples on the closed unit disk and on the unit circle in the complex plane.

Suppose that $a$ and $b$ are complex numbers with $|a|^{2}=|b|^{2}+1$. The fractional linear transformation $f(z)=(a z+b) /(\bar{b} z+\bar{a})$ maps the unit disk 
onto itself and maps the unit circle onto itself. The map $T(z)=(f(z))^{2}$ maps the unit circle onto itself and maps the unit disk onto itself. In each case $T$ is two-to-one. To see why, take $R(z)=f^{-1}(-f(z))$. $R$ is a fractional linear transformation which maps the unit circle to the unit circle and the unit disk to the unit disk. As a map of the unit disk to itself, $R$ has exactly one fixed point at $z=-b / a$, and this fixed point does not lie on the unit circle. What is interesting about this example is that as a map of the unit circle to itself, $R$ has no fixed points, in contrast with the examples on $[0,1]$.

\section{Constructing Symmetric Measures}

In this section we assume that $T$ is two-to-one and that $R$ is the reflection associated with $T$. As we shall not consider more than one two-to-one map at a time, this should cause no confusion. We will give conditions on $T$ which assure the existence of a probability measure $\operatorname{Pr}$ such that the system $(\Omega, \mathcal{F}, \operatorname{Pr}, T, R)$ is a symmetric measure-preserving system.

Let $\mathcal{I}_{T}$ denote the invariant $\sigma$-algebra of $T$ and let $\mathcal{I}_{T}^{\prime}=\left\{G \in \mathcal{I}_{T}\right.$ : $T(G)=G\}$. In some cases, Theorem 3 below can be used to show that $\mathcal{I}_{T}^{\prime}$ only contains the empty set, as we shall see in the next section.

Lemma 1. Suppose that $G \in \mathcal{I}_{T}^{\prime}$ and $G \neq \emptyset$. Let $\mu$ be a probability measure on $(\Omega, \mathcal{F})$ and suppose that $\mu(G)=1$. Then the set function $\nu$ defined on $\mathcal{F}$ by

$$
\nu(E)=\frac{1}{2} \mu\left(T\left(E \cap G \cap \Omega_{l}\right)\right)+\frac{1}{2} \mu\left(T\left(E \cap G \cap \Omega_{r}\right)\right)
$$

is a probability measure on $(\Omega, \mathcal{F})$ with $\nu(G)=1, \nu \circ T^{-1}=\mu$ and $\nu \circ R^{-1}=\nu$.

Proof. It is clear that $\nu$ is well-defined and non-negative, since $T$ carries elements of $\mathcal{F}$ to elements of $\mathcal{F}$. Next note that $T\left(G \cap \Omega_{l}\right)=T\left(G \cap \Omega_{r}\right)=G$, so $\nu(G)=1$, and that since the restriction of $T$ to each of $\Omega_{l}$ and $\Omega_{r}$ is one-to-one, $\nu$ is countably additive. Hence $\nu$ is a probability measure on $\mathcal{F}$.

Note that $R(G)=R^{-1}(G)=R^{-1}\left(T^{-1}(G)\right)=(T \circ R)^{-1}(G)=T^{-1}(G)=$ $G$, and $R\left(\Omega_{l}\right)=\Omega_{r}$, so $\nu \circ R^{-1}=\nu$.

Finally we show that $\nu \circ T^{-1}=\mu$. First observe that for any set $E \in \mathcal{F}$ we have

$$
T\left(T^{-1}(G \cap E) \cap \Omega_{l}\right)=G \cap E=T\left(T^{-1}(G \cap E) \cap \Omega_{r}\right) .
$$

To see why, recall that $T$ maps $G$ onto $G$. Therefore

$$
T\left(T^{-1}(G \cap E)\right)=G \cap E .
$$


Therefore, $g \in G \cap E$ if and only if there is some $g^{\prime} \in T^{-1}(G \cap E)$ such that $T\left(g^{\prime}\right)=g$. Now, $g^{\prime} \in T^{-1}(G \cap E)$ if and only if $R\left(g^{\prime}\right) \in T^{-1}(G \cap E)$. Since either $g^{\prime} \in \Omega_{l}$ and $R\left(g^{\prime}\right) \in \Omega_{r}$ or vice versa, $T$ maps both $T^{-1}(G \cap E) \cap \Omega_{l}$ and $T^{-1}(G \cap E) \cap \Omega_{r}$ onto $G \cap E$, as claimed.

Therefore, for any $E \in \mathcal{F}$,

$$
\begin{aligned}
2 \nu\left(T^{-1}(E)\right) & =2 \nu\left(T^{-1}(E) \cap G\right) \\
& =2 \nu\left(T^{-1}(E \cap G)\right) \\
& =\mu\left(T\left(T^{-1}(G \cap E) \cap \Omega_{l}\right)\right)+\mu\left(T\left(T^{-1}(G \cap E) \cap \Omega_{r}\right)\right) \\
& =2 \mu(G \cap E) \\
& =2 \mu(E),
\end{aligned}
$$

which finishes the proof of the lemma.

Lemma 2. Suppose that $G \in \mathcal{I}_{T}^{\prime}$ and $G \neq \emptyset$. Let $\mu$ be a probability measure on $(\Omega, \mathcal{F})$ and suppose that $\mu(G)=1$. There is a sequence $\mu_{n}$ of $R$-invariant probability measures on $(\Omega, \mathcal{F})$ such that $\mu_{n}(G)=1$ and $\mu_{n} \circ T^{-1}=\mu_{n-1}$ for $n=1,2, \ldots$.

Proof. We give a recursive construction.

Put $\mu_{0}=\left(\mu+\mu \circ R^{-1}\right) / 2$. Since $R \circ R$ is the identity map on $\Omega, \mu_{0}$ is $R$-invariant. Since $R^{-1}(G)=G$ we have $\mu_{0}(G)=1$.

Suppose now that $n$ is a positive integer and $\mu_{0}, \ldots, \mu_{n-1}$ have been constructed to satisfy Lemma 2 . Define $\mu_{n}$ by

$$
\mu_{n}(E)=\frac{1}{2} \mu_{n-1}\left(T\left(E \cap G \cap \Omega_{l}\right)\right)+\frac{1}{2} \mu_{n-1}\left(T\left(E \cap G \cap \Omega_{r}\right)\right) .
$$

Then Lemma 1 shows that $\mu_{n}$ satisfies the conditions of Lemma 2 as well.

Theorem 3. Suppose that $\Omega$ is a compact metric space, that $\mathcal{F}$ is the Borel sigma algebra and that $T$ and $R$ are continuous. Suppose that $G \in \mathcal{I}_{T}^{\prime}$ and $G \neq \emptyset$. Then there is a probability measure $\operatorname{Pr}$ on $(\Omega, \mathcal{F})$ having $\operatorname{Pr}(G)=1$ which is invariant under both $T$ and $R$. Furthermore, if $R$ has at most one fixed point and $T$ and $R$ have no fixed points in common, then $\operatorname{Pr}$ is nonatomic.

Proof. Let $\mu_{n}$ be the sequence of measures constructed in Lemma 2. Put $\sigma_{n}=n^{-1}\left(\mu_{0}+\cdots+\mu_{n-1}\right)$ for $n=1,2, \ldots$ Each $\sigma_{n}$ is invariant under $R$ and $R$ is continuous, so any limit point of the sequence $\sigma_{n}$ will also be invariant under $R$. Since

$$
\sigma_{n}=n^{-1}\left(\mu_{n} \circ T^{-n}+\mu_{n} \circ T^{-n+1}+\cdots+\mu_{n} \circ T^{-1}\right)
$$


it is easy to show that any limit point of the sequence $\sigma_{n}$ will also be $T$ invariant. (See Theorem 6.9 of Walters [1982] for the case of Borel measures on $[0,1]$.)

Now suppose that $R$ has at most one fixed point and $R$ and $T$ have no fixed points in common. We first show that no periodic point of $T$ may be an atom of Pr. Suppose that $\omega$ is a periodic point of $T$ with period $n$. Let $p=\operatorname{Pr}(\{\omega\})>0$. Note that the inverse image of an atom under $T$ is never empty, and therefore, contains either 1 or 2 points. Observe that

1. $\omega \in T^{-n}(\omega)$;

2. $T^{-n}(\omega)$ contains $\omega$ and at least one other point, and has probability $p$.

3. Each element of $T^{-n}(\omega)$ is an atom, and these atoms each have a probability which is less than $p$.

Therefore $p>0$ is not possible, meaning there are no periodic atoms.

Now we show that no non-periodic point may be an atom either. Begin with the purported atom $\omega$. For each positive integer $n$ the elements of $T^{-n}(\omega)$ are atoms, and since no atom is a periodic point, the sets $T^{-n}(\omega), n=1,2, \ldots$ are disjoint. Since these sets all have the same probability, they must have probability 0 which contradicts our assumption that $\omega$ is an atom.

Corollary 4. Suppose that $T:[0,1] \rightarrow[0,1]$ is a continuous, onto, unimodal map with $T(0)=0=T(1)$. Then there is a non-atomic probability measure on the Borel sets of $[0,1]$ and a continuous reflection $R$ of $[0,1]$ so that $([0,1], \mathcal{B}, \operatorname{Pr}, T, R)$ is a symmetric measure-preserving system.

\section{Applications}

Next we will show how Theorem 3 can be used to analyze the behavior of some symmetric unimodal maps of $[0,1]$ to itself.

Lemma 5. Let $\mathcal{I}$ be a closed bounded interval, let a be the left endpoint of $\mathcal{I}$ and let $b$ be in the interior of $\mathcal{I}$. Suppose $f: \mathcal{I} \rightarrow \mathcal{I}$

1. is continuous;

2. satisfies $f(x)>x$ on $(a, b]$;

3. satisfies $f(f(b))>a$.

Then for each $y \in(a, b]$ there is some integer $k \geq 2$ for which $f^{(k)}(b)>y$. 
Proof. Suppose not. Then for each positive integer $k$ we have $y \geq f^{(k+1)}(b)=$ $f\left(f^{(k)}(b)\right)>f^{(k)}(b)$ so $p \equiv \lim _{k \rightarrow \infty} f^{(k)}(b) \in\left(f^{(2)}(b), y\right] \subset(-a, b]$ is a fixed point of $f$. This contradicts our assumption that $f$ has no fixed points in $(a, b]$.

Theorem 6. Suppose that $([0,1], \mathcal{B}, \operatorname{Pr}, T, R)$ is a symmetric measure-preserving system and that

1. Pr has no atoms;

2. $T$ is unimodal with turning point $m$;

3. $T(x)>x$ on $(0, m]$;

4. $T(0)=T(1)=0$.

Then for any $a \in[0,1]$, if $T(a)<1$ then $\operatorname{Pr}([T(a), 1])>0$.

Proof. Suppose not. Then $\operatorname{Pr}([0, T(a)])=1$. We will use Lemma 5 to derive a contradiction. It is sufficient to examine the case $a \in(0, m]$.

Note that since $\operatorname{Pr}$ and $T$ are both invariant under $R$ and $R$ is self-inverse, $\operatorname{Pr}(A)=0$ implies $\operatorname{Pr}(T(A))=0$. Since $T$ is continuous and maps both 0 and 1 to 0 , and $\operatorname{Pr}([T(a), 1])=0$, for every $k \geq 1$ we have $\operatorname{Pr}\left(\left[0, T^{(k+1)}(a)\right]\right)=0$. From Lemma 5 for some such $k$ we have $T^{(k+1)}(a)>a$ so $[0, a] \subset\left[0, T^{(k+1)}(a)\right]$. This implies $\operatorname{Pr}([0, a])=0$, which in turn implies $\operatorname{Pr}([0, T(a)])=0$, which is our contradiction.

Corollary 7. Suppose that $m \in(0,1)$ and

1. $T:[0,1] \rightarrow[0,1]$ is unimodal with turning point $m$;

2. $T(x)>x$ on $(0, m]$;

3. $T(0)=T(1)=0$;

4. $T(m)<1, T(T(m))>0$.

Then $\mathcal{I}_{T}^{\prime}$ contains only the empty set.

Proof. Suppose not. We will now apply Theorem 3. Let Pr be the probability measure on the Borel subsets of $[0,1]$ which is preserved by both $R$ and $T$. Note that $\operatorname{Pr}$ is not atomic as $m$ is the only fixed point of $R$ and $m$ is not a fixed point of $T$. Hence $\operatorname{Pr}([T(m), 1])=\operatorname{Pr}\left(T^{-1}([T(m), 1])\right)=\operatorname{Pr}(\{m\})=0$. This contradicts Theorem 6 .

Next we consider the question of when symmetric measure-preserving systems are isomorphic. Following Walters [1982] we say that two symmetric measure-preserving systems $\left(\Omega_{i}, \mathcal{F}_{i}, \operatorname{Pr}_{i}, T_{i}, R_{i}\right), i=1,2$ are isomorphic if there exist $M_{i} \in \mathcal{F}_{i}$ with $\operatorname{Pr}_{i}\left(M_{i}\right)=1$ for $i=1,2$ such that 
(a) $T_{i}\left(M_{i}\right) \subset M_{i}$ for $i=1,2$;

(b) There is an invertible measure-preserving transformation $\Phi: M_{1} \rightarrow M_{2}$ with

$$
\begin{gathered}
\Phi\left(T_{1}(\omega)\right)=T_{2}(\Phi(\omega)) \\
\Phi\left(R_{1}(\omega)\right)=R_{2}(\Phi(\omega))
\end{gathered}
$$

for all $\omega \in M_{1}$.

Recall the symmetric tent map system, $([0,1], \mathcal{B}([0,1]), \lambda, \tau, \rho)$, defined in the introduction. Here is a formalization of the situation described in the introduction.

Theorem 8. Suppose that $T:[0,1] \rightarrow[0,1]$ is a continuous unimodal map with turning point $m, T(0)=T(1)=0, T(m)=1$, and reflection $R$. Suppose that $([0,1], \mathcal{B}, \operatorname{Pr}, T, R)$ is a symmetric measure-preserving system and the distribution function of $\operatorname{Pr}$ is a homeomorphism of $[0,1]$ onto $[0,1]$. Then $([0,1], \mathcal{B}, \operatorname{Pr}, T, R)$ is isomorphic to $([0,1], \mathcal{B}, \lambda, \tau, \rho)$.

Since the key in this theorem is having the distribution function of $\mathrm{Pr}$ be an increasing function, the following corollary to Theorem 6 is of interest.

Corollary 9. Suppose that $([0,1], \mathcal{B}, \operatorname{Pr}, T, R)$ is a symmetric measure-preserving system and

1. Pr has no atoms;

2. $T$ is unimodal with turning point $m$;

3. $T(m)=1$ and $T(0)=T(1)=0$;

4. For every interval $I \subset[0,1]$ there is some positive integer $k$ so that $m \in T^{(k)}(I)$.

Then the distribution function of $\operatorname{Pr}$ is a homeomorphism of $[0,1]$ onto $[0,1]$. In particular, $([0,1], \mathcal{B}, \operatorname{Pr}, T, R)$ is isomorphic to $([0,1], \mathcal{B}, \lambda, \tau, \rho)$.

Proof. Suppose not. Note that we must have $T(x)>x$ on $(0, m]$. Let $F$ denote the distribution function of Pr. Then for some $0 \leq a<b \leq 1$ we have $F(a)=F(b)$, so $\operatorname{Pr}([a, b])=0$. Let $I$ denote $[a, b]$, and choose $k$ so that $m \in T^{(k)}(I) \equiv I_{k}$. Note that since $T$ is continuous $I_{k}$ is a closed interval, and $I_{k}$ has probability 0 . It is also clear that $I_{k}$ has non-empty interior. Let $J_{k}=I_{k} \cup R\left(I_{k}\right) . J_{k}$ is a closed interval with probability 0 which contains $m$ 
in its interior. Hence $T\left(J_{k}\right)$ is an interval of probability 0 with right endpoint 1 and non-empty interior. This contradicts Theorem 6 .

We are, however, in a position to assert the existence of symmetric measurepreserving systems. Using Corollary 4 and the idea of the proof of Corollary 9 , it is easy to see

Theorem 10. Suppose

1. $T:[0,1] \rightarrow[0,1]$ is continuous;

2. $T$ is unimodal with turning point $m$;

3. $T(m)=1, T(0)=T(1)=0$;

4. For every interval $I \subset[0,1]$ there is some positive integer $k$ so that $m \in T^{(k)}(I)$.

Then there is a continuous reflection of $[0,1]$, denote it by $R$, and non-atomic probability measure $\operatorname{Pr}$ on $\mathcal{B}$ which assigns positive probability to all intervals, such that $([0,1], \mathcal{B}, \operatorname{Pr}, T, R)$ is a symmetric measure-preserving system which is isomorphic to $([0,1], \mathcal{B}, \lambda, \tau, \rho)$.

Condition 4 in the theorem is satisfied in many cases. See the discussion of homtervals and stable periodic orbits in Collet and Eckmann [1980].

\section{Symmetry in $([0,1], \mathcal{B}, \operatorname{Pr})$}

Suppose that we are given a probability measure $\operatorname{Pr}$ on the Borel subsets, $\mathcal{B}$, of $[0,1]$. We would like to construct transformations $T$ and $R$ so that $(\Omega, \mathcal{B}, \operatorname{Pr}, T, R)$ is symmetric. We have seen that this is easily done if the distribution function of $\mathrm{Pr}$ is continuous and strictly increasing. Suppose then we only require that it be continuous.

Theorem 11. If $\operatorname{Pr}$ is a non-atomic probability measure on the Borel sets of $[0,1]$ then there is a symmetric measure-preserving system $([0,1], \mathcal{B}, \operatorname{Pr}, T, R)$.

The proof is presented as a series of lemmas. As before, put $F(t)=$ $\operatorname{Pr}([0, t]) . \quad$ Put $F^{-1}(y)=\sup \{x: F(x) \leq y\}$ and $R(t)=F^{-1}(1-F(t))$ for all $t \in[0,1]$. Then we have:

Lemma 12. There exists $\Omega_{0} \subset \mathcal{B}$ with $\operatorname{Pr}\left(\Omega_{0}\right)=1$ such that $R(R(\omega))=\omega$ for all $\omega \in \Omega_{0}$. 
Proof. We shall take $\Omega_{0}$ to be the complement of the union of all intervals where $F$ is constant. Precisely, we define

$$
\begin{aligned}
\mathcal{J}=\{[ & {[a, b] \subset[0,1]: a<b, F(a)=F(b), } \\
& x<a<b<y \text { implies } F(x)<F(a)<F(y)\}
\end{aligned}
$$

Since the elements of $\mathcal{J}$ are disjoint closed subintervals of $[0,1]$ of positive length, $\mathcal{J}$ is countable, and the union of its elements is not $[0,1]$ since each element of $\mathcal{J}$ has probability 0 . Let $\Omega_{0}$ be the complement of the union of the elements of $\mathcal{J}$. It is clear that $\operatorname{Pr}\left(\Omega_{0}\right)=1$ and that $F$ is strictly increasing on $\Omega_{0}$.

It is easy to see that for all $x \in[0,1]$ we have $F\left(F^{-1}(x)\right)=x$. What we need to know is that if $x \in \Omega_{0}$ then $F^{-1}(F(x))=x$. To see this, observe that for all $x$ we have $x \leq F^{-1}(F(x))$, so we suppose that $x \in \Omega_{0}$ and $x<$ $F^{-1}(F(x))$. However, since $F(x)=F\left(F^{-1}(F(x))\right)$, this would imply that both $x$ and $F^{-1}(F(x))$ were in $\Omega_{0}^{c}$, a contradiction.

Now it is a simple matter to check that if $x \in \Omega_{0}$ then $R(R(x))=x$.

Lemma 13. Suppose that $g:[a, b] \rightarrow[0,1]$ is monotone and continuous. Let $h=F^{-1} \circ g$. For $z \in(a, b]$ put $c_{z}=h\left(z^{-}\right)$and put $c_{a}=a$. For $z \in[a, b)$ put $d_{z}=h\left(z^{+}\right)$and put $d_{b}=b$. Then for any $z \in[a, b]$, we have $F\left(c_{z}\right)=F\left(d_{z}\right)$.

Proof. Simply observe that since $F$ and $g$ are continuous, $F\left(c_{z}\right)=g(z)=$ $F\left(d_{z}\right)$.

First we apply Lemma 13 to prove:

Lemma 14. $R$ preserves $\operatorname{Pr}$.

Proof. It is sufficient to prove that for any $b \in[0,1], \operatorname{Pr}([b, 1])=$ $\operatorname{Pr}\left(R^{-1}([b, 1])\right)$.

First notice that $F^{-1}$ is strictly increasing and continuous from the right. Since $F$ itself is non-decreasing and continuous we conclude that $R$ is nonincreasing and continuous from the left. Let $b \in[0,1]$ be given and put $t_{b}=$ $\sup (\{x: R(x) \geq b\})$. It is straightforward to check that $R\left(t_{b}\right) \geq b$ and that $R^{-1}([b, 1])=\left[0, t_{b}\right]$.

Since $\operatorname{Pr}([b, 1])=1-F(b)$ and $\operatorname{Pr}\left(R^{-1}([b, 1])\right)=\operatorname{Pr}\left(\left[0, t_{b}\right]\right)=F\left(t_{b}\right)$, it is sufficient to show that $1-F(b)=F\left(t_{b}\right)$. This is easily done by applying Lemma 13 with $g(x)=1-F(x)$ and $z=t_{b}$, and observing that $R\left(t_{b}^{+}\right) \leq b \leq$ $R\left(t_{b}\right)=R\left(t_{b}^{-}\right)$.

We now focus our attention on constructing $T$ which preserves $\operatorname{Pr}$ and which satisfies $T=T \circ R$. We omit the straightforward proof of the following:

Lemma 15. $m \equiv F^{-1}(1 / 2)$ is the unique fixed point of $R$. 
Define the function $T$ as follows:

$$
T(x)=\left\{\begin{array}{cc}
F^{-1}(2 F(x)) & \text { if } x \in[0, m] \\
F^{-1}(2(1-F(x))) & \text { if } x \in[m, 1]
\end{array}\right.
$$

Lemma 16. $T=T \circ R$

Proof. It is easy to check that for any $x \in[0,1]$ that $F(x)=1-F(R(x))$.

Suppose that $x \in[0, m]$. Then $R(x) \geq R(m)=m$ so $R(x) \in[m, 1]$. So, $T(x)=F^{-1}(2 F(x))=F^{-1}(2(1-F(R(x))))=T(R(x))$. Similarly, if $x \in[m, 1]$ then $R(x) \leq R(m)=m$ so $R(x) \in[0, m]$ and $T(x)=F^{-1}(2(1-$ $F(x)))=F^{-1}(2 F(R(x)))=T(R(x))$.

Lemma 17. T preserves Pr.

Proof. It will be sufficient to prove that for any $b \in[0,1]$ that $\operatorname{Pr}([b, 1])=$ $\operatorname{Pr}\left(T^{-1}([b, 1])\right)$.

Fix such a $b$ and put $a_{b}=\inf (\{x: T(x) \geq b\})$ and $c_{b}=\sup (\{x: T(x) \geq b\})$. Observe that $T$ is right continuous on $[0, m]$, left continuous on $[m, 1]$, and $T(m)=1$. Therefore $a_{b} \leq m \leq c_{b}$ and $T^{-1}([b, 1])=\left[a_{b}, c_{b}\right]$. Once we show that $F(b)=2 F\left(a_{b}\right)$ and $F(b)=2\left(1-F\left(c_{b}\right)\right)$ we will be done, since averaging these equations gives $F(b)=F\left(a_{b}\right)+1-F\left(c_{b}\right)$, which in turn shows

$$
\begin{aligned}
\operatorname{Pr}([b, 1]) & =1-F(b)=1-\left[F\left(a_{b}\right)+1-F\left(c_{b}\right)\right] \\
& =F\left(c_{b}\right)-F\left(a_{b}\right)=\operatorname{Pr}\left(\left[a_{b}, c_{b}\right]\right) .
\end{aligned}
$$

(Note the use of our assumption that Pr is non-atomic.)

To see that $F(b)=2\left(F\left(a_{b}\right)\right)$ apply Lemma 13 with $g(x)=2 F(x)$ on $[0, m]$ and $z=a_{b}$, and to see that $F(b)=2\left(1-F\left(c_{b}\right)\right)$ apply Lemma 13 with $g(x)=2(1-F(x))$ on $[m, 1]$ with $z=c_{b}$.

\section{References}

[1] P. Collet and J. P. Eckmann, Iterated Maps on the Interval as Dynamical Systems, Birkhäuser, Boston, 1980.

[2] W. Rudin, Real and Complex Analysis, Third Edition, McGraw-Hill Book Company, New York, 1987.

[3] P. Walters, An Introduction to Ergodic Theory, Springer-Verlag, New York, 1982. 